had three or four loose motions daily. The spleen was slightly enlarged; the heart and lungs appeared to be normal. On Jan. 12th his temperature was $100.4^{\circ}$ in the morning and $105^{\circ}$ in the evening; on the 13 th it was $100.4^{\circ}$ in the morning and $101 \cdot 6^{\circ}$ in the evening. On the 14 th he complained of severe pain in the right side aggravated by movement and by taking a deep breath. A friction rub was heard at the inferior angle of the right scapula on auscultation. There was no dulness on percussing the base of the lung. The lower border of the liver was tender on palpation. On the previous day he had had six loose, light yellow motions. On this date he passed about one ounce of bright blood from the bowel. The tongue was dry and brown in the centre. His blood gave incomplete reaction to Widal's test; distinct clumping occurred, but many bacilli remained actively motile at the end of half an hour. His temperature was $101.2^{\circ}$ in the morning and $100^{\circ}$ in the evening. On the 15th he seemed much better; the pain in the side was relieved, there was no diarrhoea, and the tongue was cleaner and moist. His temperature was $102.2^{\circ}$ in the morning and $103.8^{\circ}$ in the evening. On the 16 th there was a second hæmorrhage from the bowel, about five ounces of bright blood being passed. There was still pain in the right side on coughing, the respiratory murmur was very feeble, and the percussion note over the extreme base posteriorly was dull. The facies was now very suggestive of enteric fever, the tongue was brown and dry in the centre, and red at the tip and edges. The abdomen was somewhat distended. There were no rose spots. The blood gave a marked but incomplete reaction to Widal's test. The blood was examined for malarial parasites, but only one degene. rated pigmented spherical body was found. His temperature was $100^{\circ}$ in the morning and $102^{\circ}$ in the evening. On the 17 th he was restless and had passed a bad night. His temperature was $101.6^{\circ}$ in the morning and $103^{\circ}$ in the evening. His pulse was 140 and his respirations were 48 per minute. His pulse was very weak; his tongue was brown in the centre, furred at the sides, and red at the tip and edges; his abdomen was more distended and there were no rose spots; a small motion was passed containing some dark altered blood and some bright blood. On the 18th his condition improved his temperature was $100.4^{\circ}$ in the morning and $104.6^{\circ}$ in the evening. On the 19th he complained of abdominal pain and was perceptibly weaker; one motion was passed containing altere blood; his temperature was $100^{\circ}$ in the morning and $102 \cdot 6^{\circ}$ in the evening. On the 20 th hia condition was somewhat better. He passed two fluid, light yellow motions without any blood in either. The lung condition remained unchanged. His temperature was $100^{\circ}$ in the morning and $103^{\circ}$ in the evening. On the 21st the blood was again subjected to Widal's test and gave an incomplete reaction. His temperature was $99.6^{\circ}$ in the morning and $1028^{\circ}$ in the evening. On the $22 \mathrm{nd}$ his temperature was $99.4^{\circ}$ in the morning and $101 \cdot 6^{\circ}$ in the evening. He continued to do fairly well till the 23rd when he became very restless and had two large hæmorrhages from the bowel. A sweiling was noticed in the epigastrium; it was dull to percussion and no fuctuation was detected. The urine contained bile pigment. In the evening he had two large hæmorrhages and became collapsed; the pulse was fluttering and very weak and the extremities were cold, but he rallied under treatment. His temperature was $98^{\circ} 4^{\circ}$ in the morning and $100^{\circ}$ in the evening. On the 24th he had a number of hæmor rhages from the bowel and became again collapsed, but rallied under treatment by opium and champagne. His teroperature was $97^{\circ}$ in the morning and $96.4^{\circ}$ in the evening. On the 25th he passed a large number of small "tarry" motions involuntarily; the pulse became imperceptible and the respirations shallow and sighing, and he died quietly about $10 \mathrm{~A} . \mathrm{M}$. His temperature that morning was $96^{\circ}$.

Necropsy.-The right lung weighed 16 ounces; its substance was very anæmic. It was adherent at the base to the chest wall and diaphragm. A small portion of the base was consolidated, dark-red in colour, and leathery in consistence. The left lung weighed nine ounces and was very anæmic ; it was slightly adherent at the base. The heart weighed 10 ounces ; the walls of the right ventricle were thin, pale, and extensively infiltrated with fat. The liver was much enlarged, especially the left lobe, and weighed 84 ounces. It contained five abscesses, three in the right lobe and two in the left. Two of the abscesses were as large as turkey's eggs the others were smaller. One of the abscess cavities con tained bile, the others contained thin yeliow pus. The liver was adherent to the diaphragm. The spleen weighed eight ounces. The kidneys were very anæmic. The stomach and small intestine showed no abnormal appearances. Close to the ileo-cæcal valve and on it were a number of irregularly shaped ulcers, some of which were two-thirds of an inch long and others smaller. The mucous membrane of the two feet of the large intestine next the valve was congested and contained numerous ulcers, the largest being nearest the valve. Beyond a distance of six inches from the valve the ulcers were small, circular, and punched out in appearance. The mucous membrane of the remainder of the large intestine and rectum was quite healthy. There was no thickening of the walls of the bowel at any point. The mesenteric glands were swollen and soft.

The spleen was incised with a sterile knife and three tubes of Parietti's broth were inoculated from it. In all three tubes there grew a bacillus and a coccus, the bacteriological examination of which gave the following results. (a) The bacillus in Parietti broth produced a slight turbidity in 48 hours and a marked turbidity in 72 hours (at $90^{\circ} \mathrm{F}$.). In broth it produced turbidity in 24 hours; after a week's incubation at $90^{\circ} \mathrm{F}$. a pellicle formed. In gelatin-stab cultures there was a growth visible in 48 hours ; there was a greyish-white slow growth on the surface and a beaded growth in the substance of the gelatin. In gelatinstreak cultures there was growth visible in 48 hoursslow growth, bluish white, irregular edges; there was no liquefaction. In glucose-gelatin shake culture there was turbidity in 24 hours, with slight gas formation in from three to eight days. Sterilised milk did not coagulate in 10 days. On potato there was moist transparent growth in 24 hours (at $90^{\circ} \mathrm{F}$.) ; in a week the growth became thick and greyish. Agar streak culture gave a thick creamy-white growth. Gelatin plate culture gave a slow growth in 72 hours ; the colonies were granular and dark in the centre; there was no liquefaction. The microscopic characters of the organism were as follows: a short, thick, sluggishly motile bacillus with rounded ends. Stained by dilute carbolfuchsin some bacilli showed slightly stained central portions and deeply stained ends. Long th eat-like forms were also seen. (b) The coccus appeared to be streptococcus pyogenes from its cultivation characters.

The clinical characters of the case were at first puzzling, but latterly they were quite consistent with a diagnosis of enteric fever. Neither the clinical history nor the postmortem appearance supported a diagnosis of dysentery. I inspected the motions regularly and they never presented. any dysenteric characters. The bacillus isolated from the spleen resembled bacillus coli in most of its characters, but it did not coagulate milk, it only produced gas after from three to eight days' growth in glucose-gelatin, and at first the growth on potato resembled that of bacillus typhosus.

\section{TWO CASES OF PULSUS PARADOXUS.}

BY JOHN HAY, M.B. VICT.,

ASSISTANT MEDICAL OFFICER, MLLL-ROAD INFIRMARY, LIVERPOOL.

UNDER certain conditions the pulse becomes distinctly smaller during inspiration or completely disappears and this form of pulse is named the "pulsus paradoxus." It is not of common occurrence, though probably not so rare as is usually supposed, often being looked upnn simply as an irregularity of the pulse whilst the rhythmical character of the irregularity is not perceived. This condition is usually bilateral but is occasionally not so, an interesting case of this nature being recorded by Dr. Thomas Harris of Manchester in THE LANCET of April 22nd, 1899, p. 1072

As to the value of the sign, opinions differ greatly, but I think that we may take it that pulsus paradoxus is not of itself pathognomonic of any condition, though it cannot be denied that it does exist in perhaps its most marked form in certain cases of indurative anterior mediastino-pericarditis ; but there are other conditions in which it is sometimes present, such as pericarditis with effusion uncomplicated by mediastinitis, also in pleuritic effusion and in patients suffering from great inspiratory dyspnœa.

The following two cases illustrate to some extent (1) these. three conditions as causative agents; (2) the relief 
afforded by thoracenteses in cases due to pleural effusion; variation with respiration becoming hardly perceptible (3) the association of the pulsus paradoxus with a pulse of low tension, as pointed out by Sir William Broadbent, who drew attention to, and emphasised the fact that, the high tension pulse hardly reacts to variations "which produce extraordinary perturbation in the low tension pulse" ; and (4) the possible short duration of the paradoxical palse. This is probably due to the fact of its dependence on low arterial blood-pressure-if from some cause the blood-pressure falls in a certain case the pulse becomes paradoxical. Stimulate

during inspiration. As the arterial tension increased again the pulse resumed its normal characteristics, the pulsus paradoxus only having been observed for a period of 20 minutes.

CASE 1.-The patient, a male child, aged three years, when in perfect health was attacked by acute bronchitis. This was accompanied by slight rise of temperature, rapid respiration and pulse, the inspiration being short and catchy and the expiration being more prolonged and wheezy. His pulse though frequent remained regular in time and force and

Pulse Tracings to Illustrate the Cases.
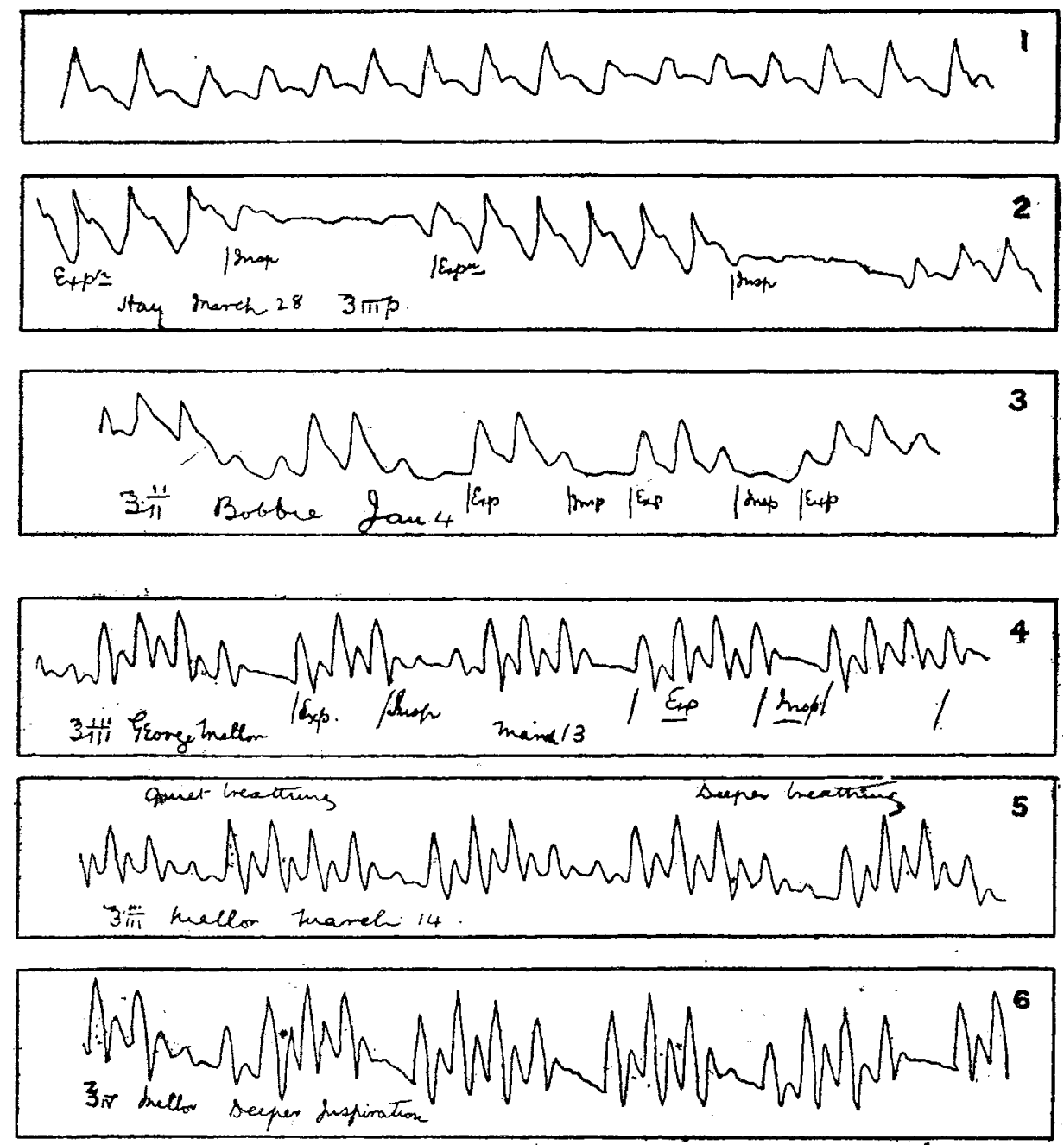

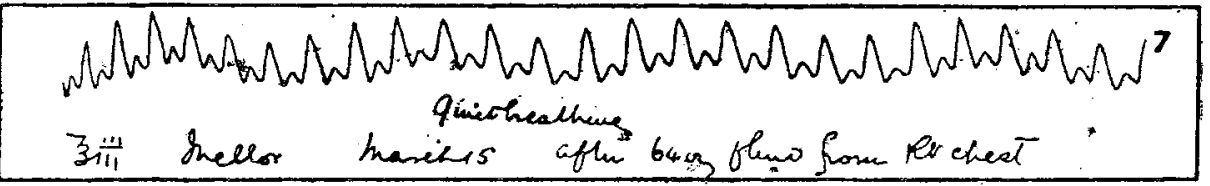

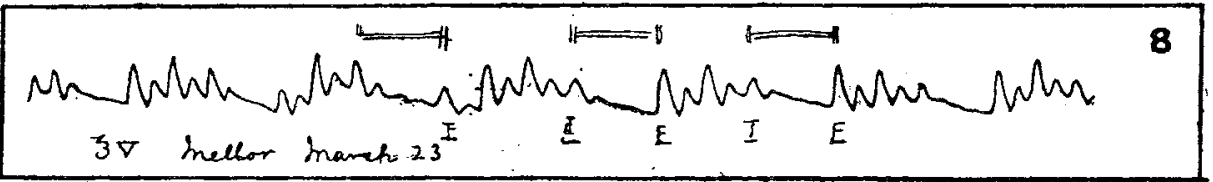

1. From normal adult, showing the effect of deep respiration. During inspiration the radial pulsation becomes more During inspiration the radial pulsation becomes more
frequent and the percussion wave not so forcible. Pressure trequent and

2. From normal adult, showing the effect of deep inspiratory efforts with closed glottis. Pressure three and a half ounces. efforts with closed glottis. Pressure three and a half ounces.
Tracing illustrating Case 1. Transient pulse condition caused Tracing illustrating Case 1. Transient pulse condition caused

4. Tracing illustrating Case 2, March 13th. Quiet breathing. Pressure three ounces.

the heart, contract the peripheral arterioles, and the pulse becomes normal in its rhythm with the increase in arterial tension. This was exemplified recently in a case necessitating venesection on account of great cyanosis due to labouring right heart, the cause being emphysema complicated by an attack of bronchitis. As the arterial tension was good and the pulse (radial) fairly strong, showing that the left ventricle was in good working condition, venesection was performed. Some 22 ounces of blood were drawn off before the man experienced relief. The radial pulse was then soft and regular in time and force and exhibited a rhythmical
5. Case 2, March 14th. Quiet breathing, but somewhat deeper than 4. Pressure three ounces.

6. Case 2, March 14th. Deeper breathing. Pressure four ounces.

7. Case 2, March 15th. Quiet breathing. Shows the effect of the withdrawal of 63 ounces of fluid from the right pleural cavity. Pressure three ounees.

8. Case 2. March 23rd. Pulsus paradoxus returned. No corresponding accumulation of fluid in the right pleural cavity. Pressure tive ounces.

of fair tension. One evening I noticed that the pulse was much softer and that during inspiration it could not be felt. I then took some pulse tracings, one of which is reproduced and which shows the relationship between the pulse and respiration ( $v i d e$ No. 3). On the next morning the pulse was again normal, being perfectly regular in time and force and not varying with respiration. The important point is that the tension was good. It was no longer a soft pulse. The respirations were somewhat less rapid, but were not markedly different from the condition of the previous evening. The convalescence was 
rapid and uninterrupted, there being no repetition of the abnormal pulse condition.

Here I think the dyspncea was the active cause, but so long as the arterial tension remained fairly good no paradoxical pulse showed itself ; however, on the occasion of the tension dropping and the pulse becoming soft, then the dyspncea was able to produce a definite effect, the result being the pulsus paradoxus.

CASE 2.-A man, aged 40 years, was admitted into hospital on Feb. 8th, 1899, suffering from broncho-pneumonia complicated by pericarditis with effusion. He had been ill for four days previously to admission. His condition was critical and he had an irregular temperature ranging between $99^{\circ}$ and $104^{\circ} \mathrm{F}$. The pulse-rate was 130 and the respirations were from 40 to 60 per minute. Under treatment he improved considerably up to Feb. 21st. From that date he siowly began to lose ground, his most prominent symptoms being marked restlessness, a very troublesome cough, considerable dyspnoea, and inability to sleep in the recumbent posture; towards the end he became delirious. His temperature remained a little above normal, the dyspncea increased, and he died suddenly on March 26th. The definite improvement in the general condition up to Feb. 21st was associated with the gradual clearing up in the lungs. Following this, however, the pericardial condition and the cardiac changes accompanying it became the main factors in the case and were the direct cause of death. On March 13th I made the following note: "The patient is still suffering greatly from dyspnoea, cannot lie down, and is occasionaliy attacked by great difficulty of breathing, the number of respirations increasing to 70 per minute. During these attacks he becomes almost frantic. They are not associated with marked cyanosis. The præcordial dulness is increased in area, extending two inches to the right and five and three-quarter inches to the left of the middle line, together making a transverse dulness of seven and threequarter inches. There is dulness in the first left interspace extending one and three-quarter inches from the middle line. No friction rub is to be felt on placing the hand over the precordium, and only an indefinite cardiac pulsation. The apex beat cannot be seen or felt, and the heart sounds are practically inaudible. There is occasionally a short systolic murmur in the fourth and fifth spaces. There are râles to be heard in the chest, more particularly over the lower lobes, and a pleural friction rub over the right middle and lower lobes. There are definite signs of fluid at the right base, the dulness reaching as high as the ninth spine. The liver is eight and a quarter inches in nipple line and is enlarged and tender. The spleen measures four inches in long diameter. The veins of the neck and arms are tense and engorged, but there is no definite cyanosis." The left pupil was dilated and larger than the right, a condition which $\mathbf{I}$ have noticed in several cases of acute pericarditis with effusion. His radial pulse was soft, frequent, and varied raythmically with the respiration.

Tracings Nos. 4, 5, 6, and 8 in the diagram show well the "pulsus paradoxus." They were, with the exception of No. 6 , taken with the patient breathing quietly. During the time that No. 6 was taken the patient was asked to breath more deeply. The five tracings all show a condition of low arterial tension, Nos. 4, 5, and 6 being distinctly hyperdicrotic. It is noticeable that where the paradoxical condition is most marked, as in No. 6, there the pulse is most hyperdicrotic.

On MIarch 15th 63 ounces of clear fluid were withdrawn from the right pleural cavity. This was followed by considerable improvement in breathing and the patient passed a comfortable night. Pulse tracing No. 7 was taken on the evening of March 15th following the thoracentesis and shows a definite improvement. There is certainly a distinct respiratory curve but otherwise the tracing might pass for that of a normal pulse of low tension.

The above alteration in the pulse was undoubtedly brought about by the removal of the fluid from the chest and although the fluid did not re-accumulate, so far as can be judged by physical signs, the paradoxical condition of the pulse returned as markedly as ever.

Tracing No. 8 was taken on March 23rd, three days before the man's death; he was breathing quietly, though rapidly. The above tracings are all from the right radial artery; others were taken from the left radial artery presenting the same condition. It was impossible to obtain any tracings from the anterior tibial artery on account of the cedema about the ankles and feet. Unfortunately no necropsy was permitted.
I would like here to express my indebtedness to Dr. Nathan Raw, as it is through his kindness that I am able to publish these cases.

\section{Clinical allotes:}

\section{MEDICAL, SURGICAL, OBSTETRICAL, AND THERAPEUTICAL.}

\section{HEMIATROPHY OF THE TONGUE.}

By L. A. PARrY, M.D., B.S. LonD., F.R.C.S. ENG., LATE HONORARY ANASTHETIST, NORTH-WEST LONDON HOSPITAL.

THE following case is of interest not so much because of the condition of the tongue as on account of the unusual cause to which it was due.

The patient, a man, aged about 50 years, complained that one side of his tongue was growing smaller. He had no other trouble whatever, but he could not quite understand why this should be, and so he had sought medical advice. The condition present was simply atrophy of one side of the tongue. The only point in his personal history bearing on the case was that some years previously he had had a serious injury to his head, resulting in fracture of the base of the skull. Now, the causes of an unilateral atrophy of the tongue are disease of the hypoglossal nucleus or disease of the same nerve. Supranuclear disease is not accompanied by wasting, or, at any rate, only to the slightest extent. Facial hemiatrophy is sometimes accompanied by hemiatrophy of the tongue, but here this need not be discussed, for there was no bemiatrophy of the face. Nuclear disease is almost always bilateral and is generally accompanied by disease of adjacent nuclei, giving rise to labial paralysis. But every now and then in chronic degenerations, such as locomotor ataxia or progressive paralysis, of the insane, this affection is unilateral. Here, too, other nuclei are generally affected and other symptoms characteristic of the disease (tabes or progressive paralysis) are present. If the lesion is below the nucleus it may be (1) within the medulla, and then there is paralysis of the leg and arm of the other side; or (2) outside the medulla, then the nerve may be damaged within the skull by meningitis or new growths, or outside the skull by wounds, cellulitis, tumours, neuritis, or caries of the highest cervical vertebræ. There are no symptoms in this case of tumours, meningitis, caries, and so on, and I think this hemiatrophy is due to injury of the nerve in its passage through the anterior condyloid foramen at the time of the fracture of the base of the skull.

Hove, Sussex.

\section{OCULAR PHENOMENA ASSOCIATED WITH CHEYNE-} STOKES RESPIRATION.

By G. Young KaLes, M.R.C.S. ENG., L.R.C.P. aND L.M. EDIN.,

LA'TE ASSTSTANT DEMONSTRATOR OF AYATOMY, ST, GEORGE'S HOSPITAI, S.W.

THE following case is, I think, worthy of record inasmuch as the ocular phenomena observed, so far as I have been able to ascertain, have not hitherto been placed on record.

A man, aged 61 years, short, dark, and thick-set, came under my observation two and a half years ago, when I found him to be suffering from a mitral systolic murmur, considerable hypertrophy of the left ventricle, and some amount of albuminuria. Dilatation of the ventricle gradually ensued and this was followed by pulmonary and hepatic congestion, increase of the albuminuria, and, finally, general anasarca, so that it may be said that the patient was more or less waterlogged for the last two years of his life. Eight weeks before his death uræmic convulsions set in and continued off and on down to ten days before the end, when he became suddenly hemiplegic, after which the convulsions ceased. The patient was now paralysed on the left side and had in addition loss of control over the sphincters. Three days before his death 